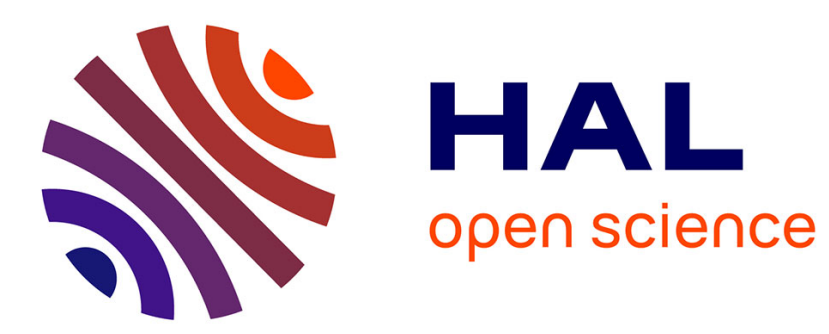

\title{
Hicksian Traverse Revisited: Conditions for the Energy Transition
}

\author{
Adrien Nguyen-Huu, Antonin Pottier
}

\section{To cite this version:}

Adrien Nguyen-Huu, Antonin Pottier. Hicksian Traverse Revisited: Conditions for the Energy Transition. 2019. hal-01966173v2

\section{HAL Id: hal-01966173 \\ https://hal.science/hal-01966173v2}

Preprint submitted on 8 Feb 2019

HAL is a multi-disciplinary open access archive for the deposit and dissemination of scientific research documents, whether they are published or not. The documents may come from teaching and research institutions in France or abroad, or from public or private research centers.
L'archive ouverte pluridisciplinaire HAL, est destinée au dépôt et à la diffusion de documents scientifiques de niveau recherche, publiés ou non, émanant des établissements d'enseignement et de recherche français ou étrangers, des laboratoires publics ou privés. 


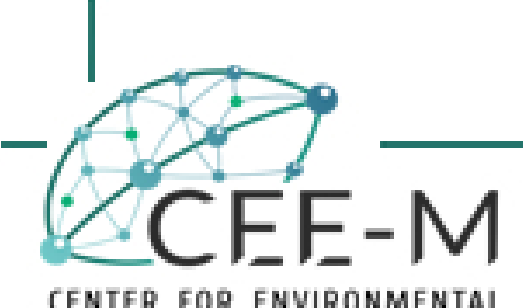

ECONOMICS - MONTPELLIER

\section{Hicksian Traverse Revisited: Conditions for the Energy Transition}

Adrien Nguyen Huu,

$\&$

Antonin Pottier

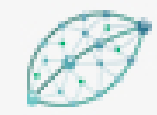

CEE-M Working Paper 2019-01 


\title{
Hicksian Traverse Revisited: Conditions for the Energy Transition*
}

\author{
Adrien Nguyen-Huu ${ }^{\dagger}$ and Antonin Pottier ${ }^{\ddagger}$
}

This version: December 27, 2018

\begin{abstract}
In this article, we analyze the transition dynamics, what Hicks called the traverse, from one equilibrium toward another one-and the conditions for such a transfer - in a bi-sectoral economy under technological shocks.

To this end, we revisit the Hick Traverse model and add to it intersectoral relations in the form of intersectoral consumption of energy for both the energy and the manufacturing sector. We investigate two distinct assumptions about consumption behavior of manufactured goods. We show that our model extends Hicks' one and leads to the same condition for a good traverse path, independently from the net energy return. We highlight that energy production technologies nevertheless provide constrains on viable states of the economy and its maximal growth rate, and that energy consumption technologies impact investment and prices crucially.
\end{abstract}

Keywords: Hicks Traverse; fixed production technology; equilibrium analysis; traverse analysis; energy economy; sectoral economy.

${ }^{*}$ Both authors thank the Energy \& Prosperity Chair for support. First author would like to acknowledge also the financial support of Agence Nationale de la Recherche (MeETMaDyS project) and the Labex Entreprendre.

${ }^{\dagger}$ CEE-M, Univ Montpellier, CNRS, INRA, Montpellier SupAgro, Montpellier, France.

${ }_{\ddagger}^{\ddagger}$ EHESS, Paris, France 


\section{Introduction}

Energy transitions - provided their usual yet diverse definitions, e.g. in SMIL (2010) - arise as a mitigation response to the climate change threat, or resource scarcity anticipation, by shifting energy production from fossil energy sources (oil, gas and coal) toward low carbon emitting or renewable technologies (biosourced, wind and solar and the controversial nuclear energy). Those technologies evidently do not have the same input requirements and macroeconomic variables are necessarily disrupted by the switching from the former productive structure toward the latter. Despite warning messages to urge such transitions, those switches are also expected to be very slow processes, and a shifting economy is not only modified at the targeted configuration, but also along the path towards it. This is explicitly recognized as a source of risk: transition risk (CARNEY, 2018). We are precisely interested in productivity and technical structural change and wave away many other challenges raised by energy transitions (see FAGNART and GERMAIN (2014) for a complementary approach).

The theoretical economic analysis of the path-and conditions for its possibility - between two different long-term economic equilibria is called the traverse analysis. The term was first coined in Capital and Growth by Hicks (1965). Hicks developed several models of traverse: the horizontal traverse considers two sectors that shared no input-output relations and studies the balance between two sectors as accumulation proceeds whereas the vertical traverse (HICKS, 1973) considers a chain of sectors, each being the input of the next, and studies the propagation of adjustement across this chain (see also LANDESMANN and SCAZZIERI (1993)). Hicks always considered the traverse analysis as a fundamental issue in macroeconomics and Solow (1984) famously qualified the traverse as "the hardest part of economics". More than half a century later, it is clear that the concept hardly inspired followers in economics: if the traverse models rose some discussions (e.g. HALEVI and Kriesler (1992); Hagemann and Scazzieri (2009); Henry and Lavoie (1997)), it has been left away for more sophisticated models which call for numerical runs to study the return-to-equilibrium path of the modeled economy after exogenous shocks. Unfortunately, more complex models (i.e. DSGE models) often study the return to equilibrium after a perturbation and give up a formal and detailed path of traverse (the transition between two equilibria), the conditions under which it naturally occurs and what happens if these conditions fail to hold. 
In the present article, we draw inspiration from Hicks Horizontal Traverse (HHT) model HiCKS (1965), originally designed with an investment good sector separated from a consumption good sector. They are replaced by a manufactured good sector, used for consumption and investment, and an energy sector, used for consumption and intermediate input for production. We thus add to the horizontality of sectors - only one sector can produce investment goods whereas the other one is only used for consumption-intersectoral relations. Resource scarcity is absent of our model, and the focus is rather on energy quality through technical coefficients of the sector. Our stance is to start from Hicks assumption that technological coefficients are constant: energy is thus taken as a complementary factor of production (and consumption). Subsequently, the model really stands close to input-output energy economics models, e.g. FAGnART and Germain (2016). It is then analyzed following the way HENRY and LAVOIE (1997) conducted the analysis of HHT model.

Several known results stem again from the analysis, and some novelties arise from our modifications. We show that the traverse condition is actually the same in our model and in Hicks', and does not invoke any energy technological configuration. We do, however, specify that the equilibrium state, as any state of the economy for that matter, only makes sense under strict conditions that intensively rely on the energy coefficients. We particularly comment on the impact of the energy efficiency of the economy ${ }^{1}$ on the maximal growth rate of the economy, or on consumption and prices. This is arguably the main message of our article that, in our stylised model, energy technologies only constitute the frame of possibly viable economic situations, but do not hinder the equilibrium stability ${ }^{2}$.

The remaining of the article is organized as follows. Section 2 introduces the model. The formal development only needs simple algebraic computations, so that our attention constantly remains on the interpretation of the mathematical model. We propose two alternative assumptions for consumption behaviors that are examined in parallel along the article, leading to two traverse styles: the quantity traverse and the price traverse. Those are relevant mainly to prove the robustness of the conclusions, similarly derived from both of them. Section 3 develops the static viability conditions, i.e.,

\footnotetext{
${ }^{1}$ Precise concepts such as the Net Energy Return (NER, Fagnart and Germain (2016)) or the Energy Return on Energy Invested (EROI, Murphy and Hall (2010)) will be mentioned later on.

${ }^{2}$ See Section 4.3.1 for details.
} 
criteria under which the economic quantities take meaningful values. Those conditions are an original contribution in contrast with Hicks model, and are sufficiently generic to stand true in many bi-sectoral input-output models. Section 4 details the traverse condition and the narrative around the bad or the good traverse. Eventually in Section 5, we give a glimpse at the model when we relax the key assumption of consumption-production equalization. We allow for wastes in one or the other sector in order to ensure convergence toward the equilibrium even in the bad traverse situation.

\section{The traverse model}

We first describe the production relations of the economy (2.1). We then specify the consumption behaviour, which is necessary to get a close accumulation model and thus the evolution of the economy (2.2).

\section{$2.1 \quad$ Production}

We consider a closed economy composed of two sectors. The first sector produces a manufactured good $M$, used indifferently for investment $\left(I_{m}\right)$ or consumption $\left(C_{m}\right)$, and is fundamental in the sense that productive capital used in both sectors accumulates through investment of this good: $\dot{K}=I_{m}$. We assume no depreciation for that good. The unit price is the same for investment or consumption: $p_{m}$.

The second sector produces energy $E$, both for intermediary $\left(I_{e}\right)$ and final $\left(C_{e}\right)$ consumption, and is also fundamental in the sense that it is a necessary primary factor of production of both sectors. The unit price of energy is the same for intermediary and final consumption: $p_{e}$.

Both sectors produce thanks to three complementary factors: capital, labor and energy. Sectors share the same productive capital $K$, which can be redistributed at no cost among sectors (putty capital), they however do not have the same capital intensity in the two sectors. They also share the common labor population $L$ which is supposed to grow exogenously with a positive rate $n: \dot{L}=n L$. Additionally, each sector consumes a part of energy $E$ produced by the second sector. Intensity of usage is given by technological coefficients that are assumed to be fixed and strictly positive ${ }^{3}$, as in HICKS

\footnotetext{
${ }^{3}$ In the following, a quantity $x$ is positive if $x \geq 0$. Strict positivity will be explicated unambiguously when necessary.
} 
(1965). The explicit link with HHT is done in Section 4.3.1. The production of one unit of good $M$ needs $\eta_{m}$ units of energy $E, a_{m}$ units of capital $K$ and $\ell_{m}$ units of labor $L$. Similarly, the production of one unit of energy $E$ needs $\eta_{e}<1$ units of energy ${ }^{4} E, a_{e}$ units of capital and $\ell_{e}$ units of labor. Those factors being complementary, we can refer to the technologies in each sector by reduced (i.e. per unit of labor) coefficients

$$
k_{e}:=\frac{a_{e}}{\ell_{e}}, \quad k_{m}:=\frac{a_{m}}{\ell_{m}}, \quad \gamma_{e}:=\frac{1-\eta_{e}}{\ell_{e}}, \quad \gamma_{m}:=\frac{\eta_{m}}{\ell_{m}} .
$$

The coefficient $k_{i}$ for $i=e, m$ is a machine-intensity per worker and precludes the energy dimension. The coefficient $\gamma_{e}$ denotes the net output per worker in the energy sector, whereas $\gamma_{m}$ denotes the required energy input per worker in the manufacturing sector.

The quantities produced in the energy and the manufacturing sector are such that production factors (labor and capital) are completely used ${ }^{5}$ :

$$
\begin{aligned}
a_{m} M+a_{e} E & =K, \\
\ell_{m} M+\ell_{e} E & =L, \\
\eta_{m} M+\eta_{e} E & =I_{e},
\end{aligned}
$$

where

$$
\begin{aligned}
M & =I_{m}+C_{m}, \\
E & =I_{e}+C_{e} .
\end{aligned}
$$

The latter equation makes the assumption that all produced energy is used, either as intermediate good for production $\left(I_{e}\right)$ or as final consumption $\left(C_{e}\right)$. This is crucial, as the mere production system determines the level of final consumption, but will be relaxed in Section 5. The quantities $I_{m}, I_{e}, C_{m}$ and $C_{e}$ must all be positive to guarantee an economic meaning. We will later investigate the conditions to be so.

The equations (1)-(2)-(3) are in absolute form. We can more conveniently write them in intensive or per capita form. We introduce $m:=M / L$ the quantity of good produced per worker, $e:=E / L$ the quantity of energy

\footnotetext{
${ }^{4}$ Notice that $\eta_{e}$ is the inverse of the primary energy return on energy invested (primary EROI), as defined in FAGNART and GERMAIN (2016) for a similar model of production. To be physically viable, the EROI of the sector must be over 1, i.e., $\eta_{e}<1$.

${ }^{5}$ In that sense, HHT is also named Hicks classical traverse model
} 
produced per worker, and $c_{m}:=C_{m} / L$ the quantity of good consumed per worker, $c_{e}:=C_{e} / L$, the quantity of energy consumed per worker. We have furthermore that $\lambda_{m}:=\ell_{m} \cdot M / L=\ell_{m} m$ is the proportion of workers in the manufacturing sector, and accordingly $\lambda_{e}:=\ell_{e} . E / L=\ell_{e} e$ the proportion of workers in the energy sector. With these notations, (1)-(2)-(3) becomes in intensive form:

$$
\begin{aligned}
k_{m} \lambda_{m}+k_{e} \lambda_{e} & =k, \\
\lambda_{m}+\lambda_{e} & =1, \\
\gamma_{e} \lambda_{e}-\gamma_{m} \lambda_{m} & =c_{e} .
\end{aligned}
$$

Equation (7) simply expresses that workers allocated either to energy or manufacturing sector. Equation (8) tells that final energy consumption per capita is a surplus, equal to net output of energy per capita (that is net output of energy per worker times proportion of energy sector workers) minus intermediate consumption per capita of energy in the manufacturing sector.

Equation (6) says that the capital-to-labor ratio $k$ of the economy is an average of sectoral capital intensities, weighted by their proportion of workers. The accumulated capital is thus the cornerstone of the economy: it determines how workers are allocated among sectors. For this allocation to be feasible with a fully employed capital (i.e. that the $\lambda$ s are between 0 and 1 ), the capital-to-labor ratio $k$ must lie between the capital intensities $k_{e}$ and $k_{m}$.

Then, recalling the reduced technological coefficients, we are able to give the quantity of goods and energy produced:

$$
m \ell_{m}=\lambda_{m}=\frac{k_{e}-k}{k_{e}-k_{m}}, \quad \text { and } \ell_{e} e=\lambda_{e}=\frac{k-k_{m}}{k_{e}-k_{m}} .
$$

These expressions highlight the aforementioned condition that $k$ must lie between $k_{e}$ and $k_{m}$. This also provides the opportunity to distinguish two cases:

$$
\text { (a) } k_{e}>k_{m}, \quad(b) k_{e}<k_{m} .
$$

The case of equality is put aside, generating indeterminacy in labor allocation. As the two labor intensities are equal, it can be seen as a variation on the one good model. 
According to equation (8), this also sets the intermediate and final consumption of energy (as all produced energy is supposedly consumed):

$$
c_{e}=\frac{\gamma_{e}\left(k-k_{m}\right)+\gamma_{m}\left(k-k_{e}\right)}{k_{e}-k_{m}}=\left(\gamma_{e}+\gamma_{m}\right) \frac{k-k_{*}}{k_{e}-k_{m}}
$$

with

$$
k_{*}:=\frac{\gamma_{m} k_{e}+\gamma_{e} k_{m}}{\gamma_{m}+\gamma_{e}}
$$

Section 3 will further discuss the role of the parameter $k_{*}$. It is important to note that the production technologies in the manufacturing and energy sector set the energy consumption. Contrary to the consumption of manufactured goods, that we discuss below, energy consumption cannot be adapted by households. It is only a by-product of the production system. In section 5 , we relax the condition of total utilisation of outputs, so that, in a given state of production, energy consumption would be lower than $c_{e}$ (but not higher).

\subsection{Two alternative dynamics}

The model is almost set up: we are left with fixing $i=I_{m} / L$ or $c_{m}$. The latter closes the model, since $i=m-c_{m}$, and

$$
\dot{k}=k\left(\frac{\dot{K}}{K}-\frac{\dot{L}}{L}\right)=k\left(\frac{I_{m} / L}{K / L}-n\right)=i-k n .
$$

The capital intensity $k$ fixes the allocation of workers, hence the manufactured good produced $m$. Once we have fixed the consumption of material good per capital, this fixes the investment $i$. If capital grows at a higher rate than population, this will increase capital intensity, which would tilt production toward the more capital intensive sector. On the contrary, if capital grows at a lower rate than population, this will decrease capital intensity, which would tilt production toward the less capital intensive sector.

Specifying how $i$ (or equivalently $I_{m}$ ) relates to other variables dramatically simplifies the evolution of the economy, as it provides an explicit control of equation (12). We therefore choose in this paper to explicit the consumption behavior, which induces the level of investment and, accordingly, the evolution of capital intensity, which drives the restructuring of the economy among the two sectors. 
Two specifications of the consumption behavior are considered in turn: a total complementarity between energy and goods consumption; a partial substitution of these two items.

1. The first one corresponds to the maximization of a Leontief utility function, and the addition of a consumption technology coefficient:

$$
c_{e}=\eta_{c} c_{m}, \text { with } \eta_{c}>0
$$

This assumption is in the flavor of HHT model Hicks (1965), where no substitution is possible. Manufactured goods need energy to satisfy customers, and energy is solely used through some manufactured goods, e.g. cars and electric devices. As mentioned by BHADuRI (1975), this is a (pure) quantity traverse: prices are determined afterward, and have a purely auxiliary role. They will not be studied with this specification.

2. The second corresponds, for a representative consumer, to the maximization of a Cobb-Douglas utility function. She allocates a constant share $\alpha$ of her income $R=w L$ to material goods and fully consumes her income. Hence:

$$
p_{m} C_{m}=\alpha w L, \quad p_{e} C_{e}=(1-\alpha) w L .
$$

Now prices really enter the model and we need to introduce the wage rate $w$ and the profit rate $r$, assumed homogeneous across sectors. The income of consumers is egal to wages $w L$ and investment is equal to profit, according to the classical saving assumption (profits are fully invested, wages are fully consumed).

$$
p_{m} I_{m}=r p_{m} K, \quad p_{m} C_{m}+p_{e} C_{e}=R=w L .
$$

This model is called the price traverse.

We study these two specifications as polar cases, because reality stands most certainly in between. The rest of the study will prove the lack of disparity in results following from these specifications.

Once the model is closed with one of the above specifications, equation (12) depends on the other variables of the model. We then have a dynamic system in $k$, which may or may not reach an equilibrium value of capital, and we can express the traverse conditions. 
Mathematically speaking, studying the traverse of an economic system consists in expressing the conditions under which an equilibrium value $\bar{k}$ to equation (12) is stable ${ }^{6}$. The underlying narrative is the following. Being first in an equilibrium situation, the economy undergoes an exogenous shock on one or several parameters of the economy (e.g. the population growth rate, as in Hicks Hicks (1965) and Henry and Lavoie HENRY and LAVOIE (1997), but also technological coefficients). The equilibrium changes as new conditions impose a different stationary value for (12). As the economy is no longer at equilibrium after that event: where it will go? This is the question raised by the traverse.

The traverse is thus the process by which an economy reaches or not a new equilibrium. A traverse, if it is good, describes the convergence of the economy toward its new equilibrium; a bad traverse means the economy diverges away from it. This qualification depends on structural conditions and market forces described by (1)-(2)-(3). The mechanisms of convergence or divergence can be detailed and shed some light on how the economy restructures itself as its capital intensity varies.

\section{Static viability conditions}

This section investigates what we called static viability conditions, that is the conditions so that all economic quantities have a meaning. These conditions stem from the positivity of the labor employed in each sector, of the economic quantities $I_{m}, I_{e}, C_{m}$ and $C_{e}$, as well as of prices in the price traverse. We begin with static viability conditions that are common to the two specifications before scrutinizing these that are specific.

For labor and capital to be fully employed, equation (6) implies, as noted above, that $k$ must lie between $k_{e}$ and $k_{m}$. This condition is already necessary in Hicks HiCKs (1965).

The second condition arises from the positivity of $c_{e}$. Equation (10) expresses $c_{e}$ as a function of a constant $k_{*}$. This value is a weighted average of $k_{e}$ and $k_{m}$, with weights that solely depend on energy efficiency in the two sectors. If capital intensity takes this value $k=k_{*}$, then the net energy

\footnotetext{
${ }^{6}$ More precisely, local stability requires to study its conditions and the basin of attraction of the equilibrium. Local stability is equivalent to global stability if the equilibrium is unique, according to the unidimensionality of the economic dynamics. That is what in fact happens in the models we consider.
} 
produced just covers the needs of the manufacturing sector. If $k$ lies between $k_{e}$ and $k_{*}$, there will be a surplus of energy, so that final consumption $c_{e} \cdot L$ is positive. If however $k$ lies between $k_{*}$ and $k_{m}$, there is not enough energy to manufactured goods, so that $c_{e}$ should be negative: we cross the limit of validity of the model. The additional static viability condition is thus that the capital intensity $k$ remains between $k_{e}$ and $k_{*}$. Conversely, if $k$ is already constrained to stay between $k_{e}$ and $k_{m}$ (first static viability condition), this condition is also sufficient for $c_{e}$ to be positive. The condition $I_{e} \geq 0$ is automatically satisfied if $C_{e} \geq 0$ is, see (3).

These two conditions come only from the production side of the model. One last condition is needed to ensure $I_{m}$ to be positive. As it depends on the consumption specifications, we deal with each case separately.

\subsection{The quantity traverse}

Consider the quantity traverse, i.e., the model under specification (13). Let us define $\gamma_{c}=\eta_{c} / \ell_{m}$. Now that consumption goods need energy to be enjoyable, $\gamma_{m}+\gamma_{c}$ is the total energy input per capita involved in the consumption of goods: it includes direct energy needed to consume the good $\left(\gamma_{c}\right)$ as well as indirect energy needed to produce the good $\left(\gamma_{m}\right)$, sometimes called embodied energy or grey energy. Final energy consumption is fixed by (10), final goods consumption by (13), and capital investment by the difference between (9) and (13). We obtain

$$
i(k)=\frac{\left(\gamma_{m}+\gamma_{c}\right)\left(k_{e}-k\right)-\gamma_{e}\left(k-k_{m}\right)}{\eta_{c}\left(k_{e}-k_{m}\right)}=\frac{\left(\gamma_{m}+\gamma_{c}+\gamma_{e}\right)\left(k^{*}-k\right)}{\eta_{c}\left(k_{e}-k_{m}\right)},
$$

with a technological constant $k^{*}$ which is, as was $k_{*}$, a weighted average of $k_{m}$ and $k_{e}$ :

$$
k^{*}=\frac{\left(\gamma_{m}+\gamma_{c}\right) k_{e}+\gamma_{e} k_{m}}{\gamma_{m}+\gamma_{c}+\gamma_{e}}=\frac{\left(\gamma_{m}+\gamma_{e}\right) k_{*}+\gamma_{c} k_{e}}{\gamma_{m}+\gamma_{c}+\gamma_{e}}
$$

Investment in capital is null when capital intensity $k$ equals $k^{*}$. This value is thus the limit of capital intensity for which the manufacturing sector produces just enough goods to satisfy consumption but none for investment. If $k$ lies between $k^{*}$ and $k_{e}$, then investment becomes negative. The static viability condition for $I_{m} \geq 0$ is indeed that $k$ remains between between $k^{*}$ and $k_{m}$. 
Putting together all the static viability conditions, we obtain the viable values of $k$ as the intersection of restraining intervals: the capital-to-labor ratio must remain between $k_{*}$ and $k^{*}$ to represent a statically viable economy. Note that this interval is not empty, since that the second expression of (17) shows that $k^{*}$ is between $k_{e}$ and $k_{*}$.

In the limit case $k=k_{*}$, we saw above that no energy can be devoted to consumption, so that the consumption of manufactured goods, constrained by the consumption technology (13), is impossible. As a consequence, all produced goods are invested, and it is easy to show that the growth rate of capital is maximal at this point.

$$
g_{\max }:=\frac{i\left(k_{*}\right)}{k_{*}}=\frac{1}{\ell_{m}} \frac{\gamma_{e}}{\gamma_{e} k_{m}+\gamma_{m} k_{e}} .
$$

It is quite interesting to notice the variables that influence $g_{\max }$. The consumption technology $\eta_{c}$ has no influence on it, because, when the growth rate of capital is maximal, there is no consumption at all. It is however strongly influence by $\eta_{e}$. When $\eta_{e}$ grows ${ }^{7}$ to $1, g_{\max }$ decreases to zero. When the technology to produce energy is not efficient, capital formation is limited.

The investment per capita $i$ can then be rewritten more compactly as:

$$
i(k)=g_{\max } k_{*} \frac{k^{*}-k}{k^{*}-k_{*}}
$$

The static viability conditions exclusively build on technical coefficients: the sectoral capital intensities $k_{m}$ and $k_{e}$, the energy technology coefficients $\gamma_{e}, \gamma_{m}$ and $\gamma_{c}$. It is worth understanding clearly how energy efficiencywhether from production or consumption technology - shapes static viability.

- What if manufacture does not need energy to produce goods $\left(\gamma_{m}=0\right)$ ? The static viability bound $k_{*}$ becomes $k_{m}$, and the maximal growth rate of capital becomes $g_{\max }=1 / a_{m}$, which is precisely the value obtained in Hicks model HenRY and LAVOIE (1997).

- What influence for the energy required in energy production? Unsurprisingly, assuming that energy production requires no energy $\left(\eta_{e}=0\right)$

\footnotetext{
${ }^{7}$ e.g. when the EROI diminishes, as EROI is the inverse pf $\eta_{e}$.
} 
does not provide a particularly illuminating case (it boils down to fix $\left.\gamma_{e}=1 / \ell_{e}\right)$. In the extreme case where we assume no energy input in energy production (i.e., an infinite EROI), the economy is only sensitive on net energy production per worker.

- We understand the radical influence of the EROI by letting it approaching the limit $\eta_{e} \rightarrow 1$, so that $\gamma_{e} \rightarrow 0$. In that case, producing one unit of energy needs one unit of energy, leading to a dead end. Because the energy sector produces no net output, it is impossible to produce any manufactured good, because it requires energy. So all workers must be in the energy sector, which is only possible when $k=k_{e}$ (indeed the static viability boundary $k_{*}$ takes the value $k_{e}$, and so does $k^{*}$ ). In this case, all energy produced is used in the production process and no net output is produced, that is no consumption nor investment is possible.

- What if the manufacturing sector has high needs for energy? Letting $\gamma_{m} \rightarrow \infty$ leads to the same result as the previous case. In that case, it is impossible to produce any material goods, because this would require an immense quantity of energy that the economy cannot provide. So all workers must be employed in the energy sector, and the economic is statically viable only when its capital intensity is $k_{e}$ (in this case, both $k_{*}$ and $k^{*}$ converge to $k_{e}$, reducing the static viability domain to this single point). In both those drastic situations, the maximal growth rate of capital is zero.

- What about the consumption technology? An increasing $\eta_{c}$ (or $\gamma_{c}$ ) means that more energy is needed for a same amount of consumption of manufactured goods. It has no effect on the $k_{*}$, which is determined only by the production technologies, but changes $k^{*}$. The effect is paradoxical as it relaxes the condition from $I_{m} \geq 0$ that $k$ lies between $k_{m}$ and $k^{*}$ : indeed, when $\eta_{c}$ increases, $k^{*}$ converges toward $k_{e}$ so that the condition is void. It is worth pondering why this is the case. Remember that capital intensity of the economy determines the produced quantities in the manufactured sector and the energy sector. As such, it also fixes the energy that is to be consumed, and the amount of goods that can be disposed of, either for consumption or investment. When goods require few energy to be consumed $\left(\gamma_{c}\right.$ low), a large amount of these goods has to be consumed to use the energy that has been produced. As a consequence, there may be nothing left for investment. 
On the contrary, when goods required a lot of energy to be consumed ( $\gamma_{c}$ high), few material goods can actually be consumed, so what is left of the production has to be invested. Because consumption is difficult, investment is easy. Thus increasing $\gamma_{c}$ penalizes the consumption of goods and leaves more room for investment. A less efficient consumption technology facilitates the investment.

This particular situation is mainly the consequence of our hypothesis that produced energy has to be fully used, either by the production process or by consumption. Could the produced energy be wasted, it would be possible to reduce consumption of manufactured goods to the

benefits of investment. This possibility will be introduced in 5, and the consequences for traverse investigated.

\subsection{The price traverse}

The previously described economy dispenses itself from price adjustments, since $c_{m}$ or $i$ are independent of such prices. On the contrary, under assumption (14), it is necessary to introduce price equations. By definition

$$
\begin{aligned}
p_{m} & =\ell_{m} w+\eta_{m} p_{e}+a_{m} p_{m} r, \\
p_{e} & =\ell_{e} w+\eta_{e} p_{e}+a_{e} p_{m} r .
\end{aligned}
$$

Those two equations, plus (15), do not enable to specify their variables until $c_{m}$ is given. Let us take the energy price as numéraire, and $\rho=p_{m} / p_{e}$ and $\omega=w / p_{e}$. Then the households consumption choice are given by the following proportions:

$$
\rho c_{m}=\alpha \omega \quad \text { and } \quad c_{e}=(1-\alpha) \omega .
$$

One can already measure the strong determinacy of prices by production, as the real wage $\omega$ is fixed by $c_{e}$. Pushing further, (19)-(20) become

$$
\begin{aligned}
& \rho=\ell_{m}\left(\omega+\gamma_{m}+k_{m} \rho r\right), \\
& 1=\ell_{e}\left(\omega+\gamma_{e}+k_{e} \rho r\right) .
\end{aligned}
$$

This, with fixed $\omega$, determines $\rho$ and $r$. Investment is $m-c_{m}$. Solving the system of equations (21)-(22) gives $\rho$ and thus $c_{m}$. After algebraic manipulations, we obtain

$$
i(k)=\frac{k}{\ell_{m}\left(k_{e}-k_{m}\right)} \frac{k^{* *}-k}{k-\alpha k_{*}}
$$


with

$$
k^{* *}=\alpha k_{*}+(1-\alpha) k_{e} .
$$

Similarly to $k^{*}$ in the quantity traverse, $k^{* *}$ is a weighted average of $k_{e}$ and $k_{*}$, so it lies in between. The weights only involve the consumption behavior, whereas in the quantity traverse, they involved production and consumption technologies. The capital intensity $k^{* *}$ plays the same economic role as $k^{*}$ : it is the limit capital intensity at which investment is null. Conversely, for capital intensity $k_{*}$, the growth rate of capital is maximal and is equal to the same $g_{\max }$ (at this point, there is no consumption, so it does not depend on the consumption behavior).

Consider Case (a) $k_{e}>k_{m}$. Then $k_{m}<k_{*}<k^{* *}<k_{e}$. For the previous viability conditions, the capital intensity $k$ is between $k_{*}$ and $k_{e}$. The positivity of investment requires simply that capital intensity $k$ is below $k^{* *}$, that is between $k_{*}$ and $k^{* *}$.

In case (b), $k_{e}<k_{m}$, then $k_{e}<k^{* *}<k_{*}<k_{m}$. The positivity of investment requires that $k$ is above $k^{* *}$ or under $\alpha k_{*}$. The last possibility is ruled out because the relative price $\rho$ would then be negative.

Summarizing, we state that the economic situation $k$ is statically viable if and only if it is between $k_{*}$ and $k^{* *}$. The similarity with the quantity traverse is striking, and there is no need to sketch the singular sub-cases obtained by specific technological values: they share the same intuitions as in the previous case.

\section{Paths of traverse}

To analysis the possibility of a traverse, that is the possibility of convergence towards a new equilibrium after a change of parameters, we follows these steps for our two models.

1. We find the equilibrium value $\bar{k}$ of the dynamics (12) $\dot{k}=i(k)-k \cdot n=$ $(g(k)-n) . k$.

2. We study whether this equilibrium satisfies the static viability conditions.

3. We examine the condition under which the equilibrium is dynamically stable for (12). 
For the last step, since the capital intensity $k$ is positive, the local stability of any equilibrium point $\bar{k}$ of dynamics (12) is mathematically expressed by the condition

$$
\left.\frac{\partial g(k)}{\partial k}\right|_{k=\bar{k}}<0
$$

\subsection{The quantity traverse}

Let us come back to the quantity traverse. We are now interested in the accumulation of capital given by (12). Recalling (18), the evolution of the capital intensity $k$ is explicitly provided by

$$
\dot{k}=\left(\frac{g_{\max } k_{*}}{k} \frac{k^{*}-k}{k^{*}-k_{*}}-n\right) \cdot k
$$

with the unique equilibrium value given by

$$
\bar{k}=k^{*} \frac{g_{\max } k_{*}}{n\left(k^{*}-k_{*}\right)+g_{\max } k_{*}}=\frac{\left(\gamma_{m}+\gamma_{c}\right) k_{e}+\gamma_{e} k_{m}}{\gamma_{m}+\gamma_{c}+\gamma_{e}+n \eta_{c}\left(k_{e}-k_{m}\right)} .
$$

We investigate the conditions of static viability for this equilibrium, i.e. we verify that $\bar{k}$ is positive and lies between $k_{*}$ and $k^{*}$. For simplicity of exposition, we separate the discussion in two cases: (a) when $k_{e}>k_{m}$ and (b) when $k_{e}<k_{m}$.

Case (a) We have here $k_{e}>k^{*}>k_{*}>k_{m}$. It is thus straightforward that $\bar{k}$ is positive and $\bar{k}<k^{*}$. The last condition is $\bar{k}$ to be above $k_{*}$, which demands $n \leq g_{\max }$.

Case (b) We have here $k_{e}<k^{*}<k_{*}<k_{m}$. If we suppose $\bar{k}$ to be positive, it is clear that it will be above $k^{*}$. For it to be under $k_{*}$, it is necessary that $n \leq g_{\max }$, which ensures that the denominator of (26) is positive, and so is $\bar{k}$.

Therefore, under the two cases, the conditions for static viability of the equilibrium boil down to the single condition

$$
n \leq g_{\max }
$$

That this condition is necessary was clear from the beginning. Indeed, when $g_{\text {max }}<n$, because $\dot{k}=(g(k)-n) . k$, the capital intensity can never grow and in fact dwindles to zero at rate at least equal to $n-g_{\max }$. 
From now on, we assume that the condition $n<g_{\max }$ is fulfilled. Under which condition is the equilibrium value locally stable? The condition of dynamic stability is:

$$
\left.\frac{\partial g(k)}{\partial k}\right|_{k=\bar{k}}=g_{\max } k_{*} \frac{1}{k^{*}-k_{*}} \frac{-k^{*}}{\bar{k}^{2}}<0 .
$$

This condition boils down to $k_{*}<k^{*}$, which is true in case (a), and only in that case, thus equivalent to $k_{e}>k_{m}$. This condition is reminiscent of Hicks HiCKs (1965). We will come back to it shortly.

\subsection{The price traverse}

In the case of the price traverse, recalling (23), short computations provide the expression for equilibrium value:

$$
\bar{k}=\alpha k_{*}+(1-\alpha) \frac{k_{e}}{1+n \ell_{m}\left(k_{e}-k_{m}\right)} .
$$

To discuss static viability conditions for the equilibrium, we again consider separately case (a) and case (b).

Case (a) We have here $k_{e}>k^{* *}>k_{*}>k_{m}$. Comparing (24) and (28), it is straightforward that $\bar{k}<k^{* *}$. Having $\bar{k}$ above $k_{*}$ requires $n \leq g_{\max }$.

Case (b) We have here $k_{e}<k^{* *}<k_{*}<k_{m}$. By the same steps done in the first case, automatically $\bar{k}>k^{* *}$ and $\bar{k}$ is under $k^{*}$ only when $n \leq g_{\max }$.

In the price quantity traverse, the condition for static viability of the capital intensity is exactly the same as for the quantity traverse: that the population growth rate does not exceed the maximal capital accumulation growth rate.

The mathematical condition for dynamic stability is given by

$$
\left.\frac{\partial g(k)}{\partial k}\right|_{k=\bar{k}}=\frac{1}{\ell_{m}\left(k_{e}-k_{m}\right)} \frac{\alpha k_{*}-k^{* *}}{\left(\bar{k}-\alpha k_{*}\right)^{2}}<0 .
$$

Since $\alpha k_{*}-k^{* *}=-(1-\alpha) k_{e}$, this is equivalent to $k_{e}>k_{m}$, that is case (a). We thus have for both traverses the same conditions for static viability and dynamic stability. Let us then abandon the distinction here and analyze the traverse for both assumptions. 


\subsection{Good and bad traverse}

We now know under which condition a traverse is good: $k_{e}>k_{m}$. A good traverse is simply the convergence of the economy, given by an initial point $k$, toward the unique equilibrium value $\bar{k}$ (which value depends of course on the type of traverse model we are in). This traverse is good in the sense that the economy converges towards its equilibrium and remains statically viable in the meantime.

There is little else to say in that case since 'things' go asymptotically to equilibrium conditions.

We investigate below three aspects of the traverse. The first one is the relation with original Hicks traverse; the second shows how the equilibrium is affected by exogenous shocks on the technology; the third is a thorough description of the economic sequence happening in the case of a bad traverse (case b).

\subsubsection{Relation to Hicks traverse}

Energy conditions are surprisingly absent from the traverse condition, which is exactly the same as in Hicks, see Henry and Lavoie (1997). On the contrary, energy holds an important role in our model through static viability conditions, that are mostly expressed by the mean of $\gamma_{m}$ and $\gamma_{e}$ (and consumption behaviors), and that are absent from the original Hicks traverse model.

It is possible to retrieve Hicks model as a special case of our framework. Our model, although expressly dedicated to the introduction of energy, can be seen more theoretically as an economy with two abstract sectors that are related through production and consumption. In the Hicks model, there is no consumption of goods through the production process. So we have to set $\eta_{e}=\eta_{m}=0$. Furthermore, investment goods are fully invested in totality whereas consumption goods are fully consumed. If we set $\eta_{c}=\infty$ in the quantity traverse, or $\alpha=0$ in the price quantity traverse, households consume only energy, so that manufactured goods are fully invested.

In this limit case, the manufacturing sector (resp. energy sector) is formally equivalent to Hick's investment sector (resp. consumption sector). Our static viability conditions boils down to $k$ must lie between $k_{m}$ and $k_{e}$. The maximal growth rate is $g_{\max }=1 / a_{m}$. We therefore simply recover Hick's results. 


\subsubsection{Exogenous shocks}

To extend Hicks's analysis to our model, we provide an impact of exogenous shocks on the value of the equilibrium state $\bar{k}$. The classical traverse study emphasizes a shock on the population growth rate $n$ HENRY and LAVOIE (1997). One can easily see that the effect is strictly the same in the amended version. We add to this the effect of technological shocks, that are under the spotlight for this article. Recalling that the traverse refers to the path from a former equilibrium state to a new one, we assess the impact of a change in the technological coefficient.

A synthetic view is given by Table 4.3.2. The analysis of shocks can be resumed by the sign of partial derivatives of the equilibrium expression $\bar{k}$, since they are monotonous. Along the line of preceding results, those derivatives provide the same sign for both traverse models.

\begin{tabular}{|c||c|c|c|c|c|c|}
\hline$x=$ & $a_{m}$ & $a_{e}$ & $\ell_{m}$ & $\ell_{e}$ & $\eta_{m}$ & $\eta_{e}$ \\
\hline $\operatorname{sign}\left(\frac{\partial \bar{k}}{\partial x}\right)=$ & + & + & $-^{*}$ & $-^{*}$ & $\operatorname{sign}\left(k_{e}-k_{m}\right)$ & $\operatorname{sign}\left(k_{e}-k_{m}\right)$ \\
\hline
\end{tabular}

Table 1: sign of partial derivatives of $\bar{k}$ w.r.t. technological parameters. *: the value holds only if $n \leq 1 / a_{m}$

Results are unambiguous. An increase in $a_{i}, i=m, e$ (corresponding in a decrease in capital productivity) will increase the respective value of capital intensity $k_{i}$ of sector $i$, ceteris paribus. It will therefore shift upwards the static viability conditions, but also the equilibrium capital intensity $\bar{k}$. The converse applies for $\ell_{i}, i=m, e$, since an increase of the latter implies a decrease of $k_{i}$, ceteris paribus. The result for energy efficiency is also very intuitive: every move toward a better energy efficiency leads to an equilibrium economy that put less workers into the energy sector, which requires a capital intensity of the economy closer to the capital intensity of the manufacturing sector. Thus, an increase in $\eta_{i}, i=m, e$, which translates a decrease of efficiency, moves the equilibrium toward $k_{e}$.

\subsubsection{Bad traverses}

There are several ways for an economy to become non viable after a change in the parameters. 
(i) after the shock, the current economy finds itself in a non statically viable state. Indeed a shock in parameters modifies the viability range of capital intensity. For example, a large decrease in capital productivity could put the current capital intensity outside the viability range. In that case, the economy stops from the start.

(ii) if the current state is viable, that the good traverse condition holds, but the new equilibrium value is not statically viable. For example a large increase in population growth may drive $n$ above $g_{\max }$. In this case, the economy will converge to its new equilibrium value but will sooner or later be moved outside the viability range.

(iii) the current economy is statically viable, but the traverse is bad, i.e. $k_{e}<k_{m}$. The economy will move from its current position and cross the viable range.

In this paragraph we focus only on the last case, the bad traverse. The manufactured good is more capital intensive per capita than the energy sector. Note that in this case, the growth rate of capital $g(k)$ is an increasing function of capital intensity. According to sections 4.1 and 4.2, the equilibrium $\bar{k}$ is not attractive, and any economy away from it will diverge from it. Two economic situations are possible.

Consider a first situation with $k<\bar{k}$ : the capital intensity of the economy is below its long-term equilibrium. As a consequence, more workers are allocated to the energy sector than at equilibrium, more energy is produced, so that more manufactured goods has to be consumed in order to use the energy that has been produced. As a result, the growth rate of capital is under its equilibrium value $(n)$, so that investment is not sufficient to build capital stock for the new workers coming onto the market, so that capital intensity further dwindles $(\dot{k}<0)$. To ensure full employment, workers must deploy in the less capitalistic sector, the energy sector, which exacerbates the trend. The situation worsens until it reaches a configuration dedicated only to consumption (of goods and energy) $\left(m=c_{m}\right)$, and the economy ceases to be viable.

The second situation happens when $\bar{k}<k$. In that case, the capital intensity is above its equilibrium value. Allocation of workers is tilted towards the manufacturing sector, whose production is more than enough to cover consumption and investment to increase the capital stock. The growth rate of capital is above its equilibrium value. As a result, capital intensity grows 
$(\dot{k}>0)$, so that more workers will be led to the more capital intensive sector, the manufacturing sector. This will reduce production in the energy sector, and thus energy available for consuming goods. Investment will grow and capital intensity will increase further. In the absence of counter strength, the energy sector shrinks up to the point when it provides only enough energy for intermediate consumption ( $k$ reaches $k_{*}$ ): as this point where the investment rate is maximal, no consumption is possible because of lack of energy. At this point, the economy ceases to be viable.

\section{A flexible traverse}

Our model is inspired by how Hicks has formulated the traverse problem. With two alternative consumption assumptions, the dynamics follows from investment, which in turns is dictated by consumption behavior. It shows a dependency on one key hypothesis: the full utilization of goods produced, whether energy or manufactured goods ${ }^{8}$.

Consider the bad traverse with $k>\bar{k}$. The capital intensity keeps growing because manufactured goods are invested, although this investment puts the economy further away from equilibrium. If manufactured goods could be disposed of, in this case those which are invested should be entirely wasted: the investment rate would be zero, the capital intensity would dwindle at rate $n$ towards $\bar{k}$ and the economy would reach its equilibrium. Conversely in case $k<\bar{k}$, if energy can be wasted, then it should be. If we waste entirely the energy dedicated to consumption, no consumption of manufactured goods is necessary. All manufactured goods can then be devoted to investment so that capital intensity grows towards $\bar{k}$ and the economy is moved toward its equilibrium. We can even allow for a minimal consumption if wanted.

In Hicks' framework, with the investment sector separated from the consumption sector, how the bad traverse can be improved is slightly different. With $k>\bar{k}$, the economy diverge from its equilibrium because too many investment goods are actually invested. If investment goods were produced but not set in place, the economy would move to its equilibrium. With $k<\bar{k}$ however, there is not enough investment goods produced and restriction of consumption does not provide any leeway to increase investment, because

\footnotetext{
${ }^{8}$ It holds in a particularly straight manner for energy as its consumption follows from net surplus after intermediate consumption from productive sectors
} 
consumption goods and investment goods are different and one cannot be transformed into the other. So the bad traverse cannot be avoided.

So in our model, if we can control the quantities wasted, then we can put the economy back on its equilibrium even in the bad traverse $k_{e}<k_{m}$. There is only a trade-off between the amount of goods (manufacture or energy) wasted at each time and the time to reach the equilibrium. From the above insight, the more we are ready to waste, the faster the economy will converge to its equilibrium.

To escape the bad traverse when there is too much investment, we have seen that the alternative is between investment and waste. If we introduce $u_{m}$ the utilisation rate of the quantity of manufactured goods left for investement, the quantity really invested is $I_{m}=u_{m}\left(M-C_{m}\right)$ and the quantity wasted is $\left(1-u_{m}\right)\left(M-C_{m}\right)$. This $u_{m} \in[0,1]$ is the control of our system to avoid the bad traverse. Similarly, when there is too little investement, the alternative is to consume energy (in conjonction with manufactured goods) or to waste energy (which makes room for investment). We introduce $u_{e}$ the utilisation rate of the quantity of energy left for consumption, the quantity really consumed is $C_{e}=u_{e}\left(E-I_{e}\right)$ and the quantity wasted is $\left(1-u_{e}\right)\left(E-I_{e}\right)$.

In the remainder of the section, we discuss the two sides of the bad traverse, but only for the quantity traverse. Before we move on to the analysis, notice one last thing. In the quantity traverse, once the amount of goods wasted - whether manufactured goods or energy - has been chosen, we know all the quantities produced. The novelty, compared to section 2.2, is that the used (sold) quantities do not add up to the produced quantity. Pricing at production cost (like (19)-(20)) would imply that each sector make negative profits and that there is a part of unspent revenues. To avoid these difficulties one can assume that prices are such that the quantities sold cover the production costs, and amend the price equations (19)-(20) accordingly. This implies that a price analysis can be pursued in the quantity traverse as well as in the price traverse, and that wastes' impact on relative price can be evaluated and included in considered criterion for an optimal traverse path.

\subsection{Controlling the flow of goods toward investment}

We set ourselves in the quantity traverse, and take back the situation of a bad traverse with $k>\bar{k}$. Intending to focus on one specific task, that is, define how waste disposal can allow to converge to the new economic equilibrium $\bar{k}$, we will assume that static viability of the latter as well as the present 
economic state $k$ is satisfied, i.e., $k_{e}<k^{*}<\bar{k}<k<k_{*}<k_{m}$. To get toward $\bar{k}$, the dynamics of capital must decrease $(\dot{k}<0)$, and so investment must be lower than $n k$. We introduce the utilisation control

$$
u_{m}:=\frac{i}{m-c_{m}} \in[0,1]
$$

To follow the good traverse path, and recalling (12), we thus need $u_{m}<\frac{n k}{m-c_{m}}$. Algebraic manipulation from (25) result in

$$
u_{m}<u_{m}^{\lim }(k):=\frac{n}{g_{\max }} \frac{k}{k_{*}} \frac{k^{*}-k_{*}}{k^{*}-k} .
$$

How does this function behave? Notice that $u_{m}^{\text {lim }}$ is a convex decreasing continuous function on the interval $\left[\bar{k}, k_{*}\right]$, with $u_{m}^{\text {lim }}=n / g_{\max }$ when $k=k_{*}$. Suppose $n<g_{\max }$ (which excludes the limiting and uninteresting case of $n=g_{\text {max }}$ and hence $\bar{k}=k_{*}$ ), in this case there exists a left neighborhood of $k_{*}$ for which $u_{m}$ is constrained to be strictly lower than one: not all values of $u_{m}$ might work.

All possible utilisation rates $u_{m}$ are comprised between the $\mathrm{x}$-axis and the orange (plain) line $u_{m}^{\lim }$ on figure 1 (at the right of $\bar{k}$ ). At this stage, there is no optimal rate $u_{m}$, but only constrains on possible efficient values given by the expressions (30) and (31).

The labor population growth rate, as well as the static viability conditions $k^{*}$ and $k_{*}$, influence the spectrum of possible efficient rates, but a controlled traverse is yet manageable at any state $k \in\left[\bar{k}, k_{*}\right]$, provided ${ }^{9}$ that $n>0$. Technically, the equilibrium value $\bar{k}$ can be reached in finite time (and not only asymptotically) for all states in that interval: the lower the value of $u_{m}$, the faster the convergence. It is also not required that the control be constant during the traverse process. The choice of a specific value for $u_{m}$ depends on the cost associated to disposal of manufactured goods, which is absent from present considerations. We thus leave optimal control apart to provide all the possible controls $u_{m}$ that allow to reach the target ${ }^{10}$.

\footnotetext{
${ }^{9}$ When $n=0$, all goods dedicated to investment have to be wasted, capital stay constant; because there is no dilution of capital due to population growth, capital intensity stays constant and the equilibrium value is never reached. Allowing depreciation of capital would relax this condition.

${ }^{10}$ This recalls the principles of Viability theory (AUBIN, 2009): find all possible states and controls that satisfy constraints along the path and allow to reach a target.
} 


\subsection{Controlling the level of energy available for final consumption}

We now take the other situation where $k_{e}<k^{*}<k<\bar{k}<k_{*}<k_{m}$. If nothing can be made in Hicks traverse, cf. discussion supra, in our model we can interfere with energy availability to restore the path to equilibrium. We introduce the utilization control

$$
u_{e}:=c_{e} /\left(e-i_{e}\right) \in[0,1]
$$

Since investment is impacted positively by energy disposal, see right below, the static viability conditions remain the same as in Section 3.1, and deserve no comment.

The control has to be chosen so as to increase capital intensity. Since $k_{e}<k_{m}, \dot{k}<0$ for $u_{e}=1$, and we seek values in the unit interval such that $\dot{k}>0$. Straightforward computations provide the condition

$$
u_{e}<u_{e}^{\lim }(k)=\frac{\gamma_{c}}{\gamma_{m}+\gamma_{e}}\left(\frac{\left(1-n \ell_{m}\left(k_{m}-k_{e}\right)\right) k-k_{e}}{k_{*}-k}\right) .
$$

How does that function behave? It is quite similar to $u_{m}^{\lim }$. Provided that $n<g_{\max }$, we can verify that $u_{e}^{\lim }(k)>0$ for all statically viable states $k>k^{*}$. The function is convex increasing, verifying $u_{e}^{\lim }(\bar{k})=1$, if $\bar{k}<k_{*}$ (i.e., $\left.n<g_{\max }\right)$. All possible utilisation rates $u_{e}$ are comprised between the $\mathrm{x}$-axis and the blue (dotted) line $u_{e}^{\mathrm{lim}}$ on figure 1 (at the left of $\bar{k}$ ).

The degree of leeway in choosing $u_{e}$ thus depends also on the value of $n$. The closer $n$ is to $g_{\max }$ however, the steeper is $u_{e}^{\lim }$ at $\bar{k}$. The limit case $n=0$ must be excluded since it implies $\bar{k}=k^{*}$, and the traverse problem makes only sense for $k^{*}<k<\bar{k}$. The opposite limit case, where $n=g_{\max }$, is however not forbidden. We have $\bar{k}=k_{*}$, which implies $u_{e}^{\lim }(\bar{k})=0$. Accordingly $u_{e}^{\lim }$ is negative for all statically viable values of $k$. Therefore it is impossible to reach the equilibrium with any control. This is not surprising since the population growth is so high that it cannot be compensated by any investment policy.

As in the previous subsection, we dispense ourselves of discussing the optimality of a given rate of wasting. We only provide the set of allowed values, synthesized by the blue line in Figure 1 . 


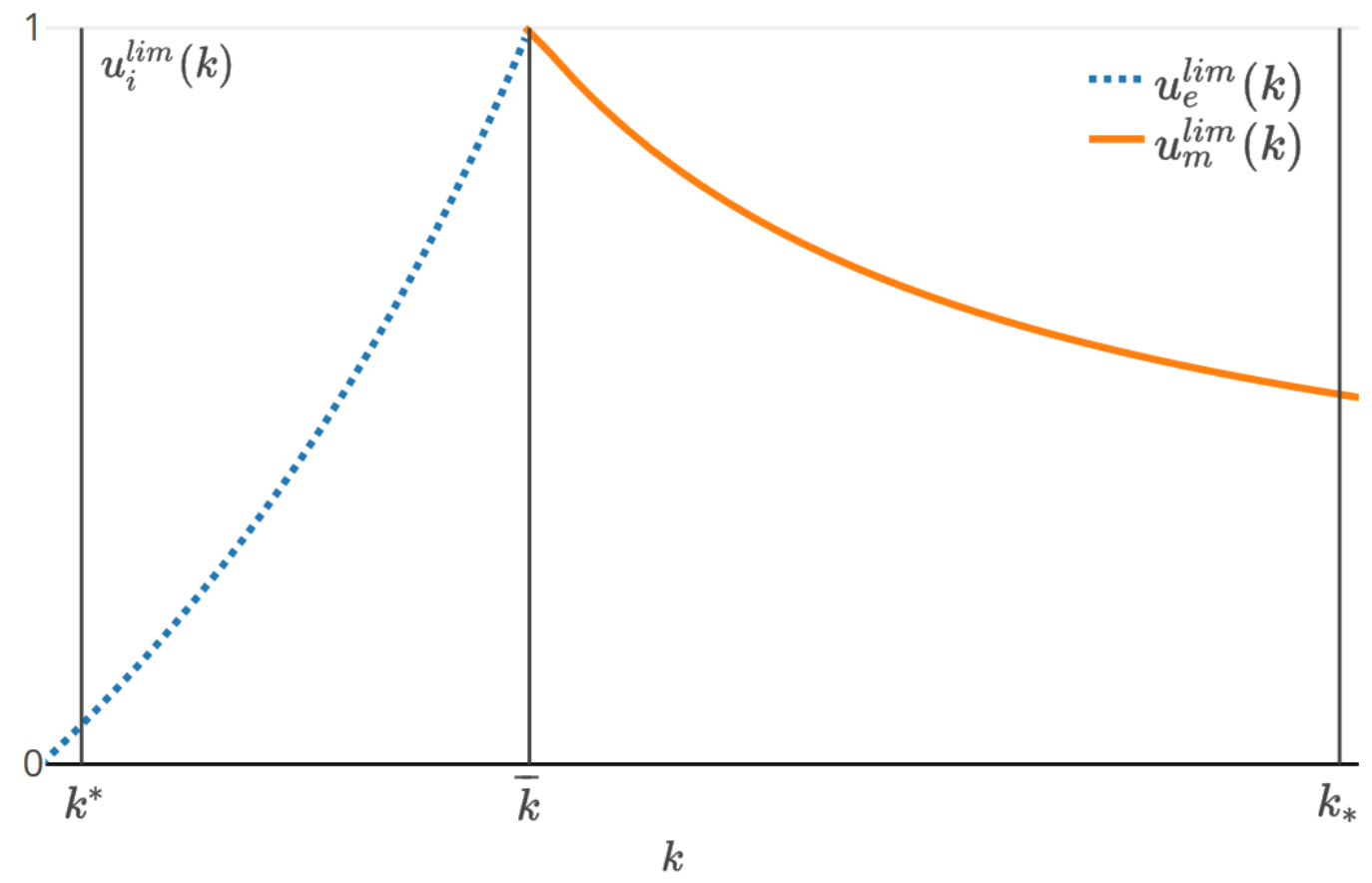

Figure 1: Illustration of the functions $u_{m}^{\lim }(k)$ (orange line) and $u_{e}^{\lim }(k)$ (blue dotted), and the efficiency area below, for an arbitrary set of parameters verifying the conditions of subsections 5.1 and 5.2.

\section{Conclusion}

The current revisited version of HHT changes its narrative to understand the influence of production technologies when energy is a fundamental factor of production. The tractability of the model serves our message, despite acknowledging deficiencies and simplifying assumptions which go along with it. We explicated the slight importance of the consumption behavior and the great importance of the full utilization of production factors in the model. In an economy where those factors do not adjust, and where households also manage a basket of two goods, we found many constrains to be satisfied (the static viability conditions) that were not present in the original traverse. Relaxing full utilization of factors may ease the process of traverse toward the desired state of the economy, as others traverse models suggest (e.g. LAVOIE and RAMirez-GASTON (1997)). We proposed an option with waste disposal, opening the door to many others which can be dealt with similarly. 
Our message is twofold. First, general messages related to the energy transition can be delivered. The transition to a (desired or required) new equilibrium need not be natural, and is only indirectly related to energy technologies. If energy does not impact the stability of the stationary state of the economy, it limits the maximum growth rate as well as all other economic quantities one seeks to monitor. Those very broad conditions should apply in many two-sectors (energy-manufacture) models.

Finally, the traverse study itself delivers an important message especially in the bad case, and especially when energy limits viable economic states. The translation of the classical Hicksian bad traverse in the energy terminology draws a fresh look at both the traverse narrative (HENRY and LAVOIE, 1997) and how an energy transition can fail or be problematic (FAGNART and Germain, 2014). One may easily play with our generic analysis to draw the scenario which seems the more relevant to him.

\section{References}

Aubin, J.-P., 2009 Viability theory. Springer.

Bhaduri, A., 1975 On the Analogy between the Quantity-and the PriceTraverse. Oxford Economic Papers 27(3): 455-461.

Carney, M., 2018 Transition in thinking and action. Speech given at International Climate Risk Conference for Supervisors, April 6.

Fagnart, J.-F. and M. Germain, 2014 Can the energy transition be smooth? CEREC Discussion Paper 201411 and FAERE Working Paper 215.04 .

Fagnart, J.-F. and M. Germain, 2016 Net energy ratio, EROEI and the macroeconomy. Structural Change and Economic Dynamics 37: 121-126.

Hagemann, H. and R. Scazzieri (Eds.), 2009 Capital, time and transitional dynamics. Routledge.

Halevi, J. and P. KRIESLER, 1992 An introduction to the traverse in economic theory. In Beyond the Steady State, pp. 225-234. London: Palgrave Macmillan. 
Henry, J. and M. Lavoie, 1997 The Hicksian traverse as a process of reproportioning: some structural dynamics. Structural Change and Economic Dynamics 8(2): 157-175.

Hicks, J., 1965 Capital and growth. Oxford: Oxford University Press.

Hicks, J., 1973 Capital and time: a neo-Austrian theory. Oxford: Oxford University Press.

Landesmann, M. A. and R. Scazzieri, 1993 Commodity flows and productive subsystems: An essay in the analysis of structural change. In The dynamics of the wealth of nations, pp. 209-245. London: Palgrave Macmillan.

Lavoie, M. and P. Ramirez-Gaston, 1997 Traverse in a Two-Sector Kaleckian Model of Growth With Target-Return Pricing. The Manchester School 65(2): 145-169.

Murphy, D. J. and C. A. Hall, 2010 Year in review-EROI or energy return on (energy) invested. Annals of the New York Academy of Sciences 1185(1): 102-118.

Smil, V., 2010 Energy transitions: history, requirements, prospects. ABCCLIO.

Solow, R. M., 1984 Mr Hicks and the classics. Oxford Economic Papers 36: 13-25. 


\section{CEE-M Working Papers' - 2019}

WP 2019 - 01: $\quad$ Adrien Nguyen-Huu. \& Antonin Pottier «Hicksian Traverse Revisited: Conditions for the Energy Transition» 